\title{
Human milk proresolving mediators stimulate resolution of acute inflammation
}

\author{
H Arnardottir ${ }^{1,2}$, SK Orr ${ }^{1,2}, \mathrm{~J} \mathrm{Dalli}^{1}$ and CN Serhan ${ }^{1}$
}

Human milk contains nutrients and bioactive products relevant to infant development and immunological protection. Here, we investigated the proresolving properties of milk using human milk lipid mediator isolates (HLMIs) and determined their impact on resolution programs in vivo and with human macrophages. HLMls reduced the maximum neutrophil numbers $\left(14.6 \pm 1.2 \times 10^{6}-11.0 \pm 1.0 \times 10^{6}\right.$ cells per exudate) and shortened the resolution interval $\left(R_{i} ; 50 \%\right.$ neutrophil reduction) by $54 \%$ compared with peritonitis. Using rigorous liquid-chromatography tandem-mass spectrometry (LC-MS-MS)-based lipid mediator (LM) metabololipidomics, we demonstrated that human milk possesses a proresolving LM-specialized proresolving mediator (LM-SPM) signature profile, containing SPMs (e.g. resolvins (Rv), protectins (PDs), maresins (MaRs), and lipoxins (LXs)) at bioactive levels (pico-nanomolar concentrations) that enhanced human macrophage efferocytosis and bacterial containment. SPMs identified in human milk included D-series Rvs (e.g., RvD1, RvD2, RvD3, AT-RvD3, and RvD4), PD1, MaR1, E-series Rvs (e.g. RvE1, RvE2, and RvE3), and $L X s$ ( $L X A_{4}$ and $\left.L X B_{4}\right)$. Of the SPMs identified in human milk, RvD2 and MaR1 (50 ng per mouse) individually shortened $R_{\mathrm{i}}$ by $\sim 75 \%$. Milk from mastitis gave higher leukotriene $\mathrm{B}_{4}$ and prostanoids and lower SPM levels. Taken together, these findings provide evidence that human milk has proresolving actions via comprehensive LM-SPM profiling, describing a potentially novel mechanism in maternal-infant biochemical imprinting.

\section{INTRODUCTION}

The acute inflammatory response is critical in infection and injury. The initiation and resolution of inflammation are important in host defense, each governed by bioactive lipid mediators (LMs) that drive the influx and function of immune cells, and eventually cell efflux and tissue repair. ${ }^{1,2}$ Newly identified families of bioactive LMs, biosynthesized from essential fatty acids (EFAs), that actively stimulate resolution of inflammation were uncovered in self-resolving exudates and their structures elucidated. ${ }^{1}$ Collectively, they are coined specialized proresolving mediators (SPMs). ${ }^{1}$ SPMs comprise several families that include arachidonic acid (AA)-derived lipoxins (LXs), eicosapentaenoic acid (EPA)-derived resolvins (RvEs), and docosahexaenoic acid (DHA)-derived resolvins $(\mathrm{RvD})$, protectins (PDs), and maresins (MaRs); these structurally distinct families are each host protective with defining actions in anti-inflammation (e.g., limit further neutrophil inflammation), proresolution (e.g., enhancing macrophage clearance of apoptotic cells, debris, and bacteria), pain reduction, and wound healing (reviewed in Serhan ${ }^{1}$ ). SPMs are evolutionarly conserved biochemical signals, as they are present in trout, salmon, and planaria (reviewed in Serhan ${ }^{1}$ ), and have already been identified in human organ systems, including plasma (RvD1, RvD5, RvD6, and RvE2), ${ }^{3}$ adipose tissue (RvD1, RvD2, PD1, RvE1, and $\left.\mathrm{LXA}_{4}\right),{ }^{4}$ placenta (RvD1, AT-RvD1, RvD2, and PD1), ${ }^{5}$ and recently human milk (RvD1, RvE1, and $\left.\mathrm{LXA}_{4}\right){ }^{6} \mathrm{LXA}_{4}, \mathrm{RvE} 1, \mathrm{RvD} 1$, and RvD2 each reduce mucosal inflammation, stimulate the innate immune response, and activate resolution of periodontal disease, colitis, and dermal inflammation. ${ }^{7-9}$

Human milk is recognized as being important for infant development, providing essential nutrients and bioactive products relevant for maternal-mucosal immune defense and immune system maturation. ${ }^{10}$ The $n$ - 3 EFA including EPA and DHA are enriched in human milk. ${ }^{11}$ For infants, and particularly premature infants, injurious and infectious insult

\footnotetext{
${ }^{1}$ Center for Experimental Therapeutics and Reperfusion Injury, Department of Anesthesiology, Perioperative and Pain Medicine, Harvard Institutes of Medicine, Brigham and Women's Hospital and Harvard Medical School, Boston, Massachusetts, USA. Correspondence: CN Serhan (cnserhan@zeus.bwh.harvard.edu)

${ }^{2}$ These authors contributed equally to this work. 
can be detrimental. ${ }^{12}$ Hence, protective mechanisms for resolving infection and inflammation in a timely manner and educating the innate immune system in early life are critical and of general interest. In this report, we present evidence for new immunoresolving properties of human milk. Using selflimited acute inflammation and LM metabololipidomics, we found that isolates from human milk contain chemical signals with proresolving actions, namely limiting neutrophil trafficking in vivo, enhancing human macrophage phagocytosis of apoptotic polymorphonuclear neutrophil (PMN) (efferocytosis), and bacterial containment. These actions were attributed to the proresolving LM-SPM signature profile of identified bioactive mediators that included D-series resolvins (ATRvD1, RvD2, RvD3, AT-RvD3, RvD4, RvD5, and RvD6), PDs (PD1 and AT-PD1), MaR1, E-series Rvs (RvE2 and RvE3), and LXs (AT-LXA ${ }_{4}$ and $\mathrm{LXB}_{4}$ ). The LM-SPM profile was altered in human milk from inflamed mammary glands (mastitis) with higher prostanoids and leukotriene $\mathrm{B}_{4}\left(\mathrm{LTB}_{4}\right)$ and lower SPM levels, and had reduced ability to accelerate resolution interval $\left(R_{\mathrm{i}}\right)$. Hence, the present results provide evidence for bioactive resolution signals in human milk that are linked to homeostasis, resolution of inflammation, and innate host responses.

\section{RESULTS}

\section{HLMIs stimulate resolution of inflammation}

To investigate whether human milk exerts proresolving actions, we used human milk chromatographic isolates with self-limited acute inflammation in vivo and mapped leukocyte trafficking. Because SPMs, including Rvs, PDs, and MaRs, stimulate resolution $^{1}$ and elute within the methyl formate chromatographic fractions from C18 solid-phase extraction, ${ }^{3}$ we obtained human milk isolates from these fractions (referred to as human milk lipid mediator isolates (HLMIs)) and assessed their ability to accelerate resolution of acute inflammation in vivo. First, selflimiting acute inflammation was initiated by intraperitoneal injection of yeast cell wall particles (zymosan, $1 \mathrm{mg}$ per mouse), and to quantitate resolution we used defined resolution parameters of acute inflammation. ${ }^{13,14}$ The self-limited response reached maximal neutrophil numbers $\left(\Psi_{\max }=14.6 \pm 1.2 \times 10^{6}\right.$ cells per murine exudate) at $12 \mathrm{~h}\left(T_{\max }\right)$, which was followed by subsequent decline (Figure 1a). Administration of HLMIs immediately before inflammatory challenge gave an $\sim 23.1 \pm 8.9 \%$ reduction in $\Psi_{\max }\left(11.0 \pm 1.0 \times 10^{6}\right.$ cells per exudate; Figure 1a, b). Reduction in neutrophil levels was observed throughout the course of inflammation resolution in mice administered HLMIs, with $31.3 \pm 4.4 \%$ and $24.5 \pm 10.9 \%$ fewer neutrophils at 24 and $48 \mathrm{~h}$, respectively, compared with peritonitis plus vehicle (Figure 1c).

To quantify the regulation of leukocyte trafficking at the site of inflammation, we investigated the $R_{\mathrm{i}}$ that quantitates the local kinetics of leukocyte infiltration, with the $R_{\mathrm{i}}$ being defined as the time interval between $T_{\max }$ and $T_{50}$ (the time interval when the number of infiltrated PMN drops to half of the peak number). ${ }^{13,14}$ We found that HLMI administration gave $54 \%$ reduction in $R_{\mathrm{i}}$ from 26 to $12 \mathrm{~h}$ (Figure 1a, b). These results a

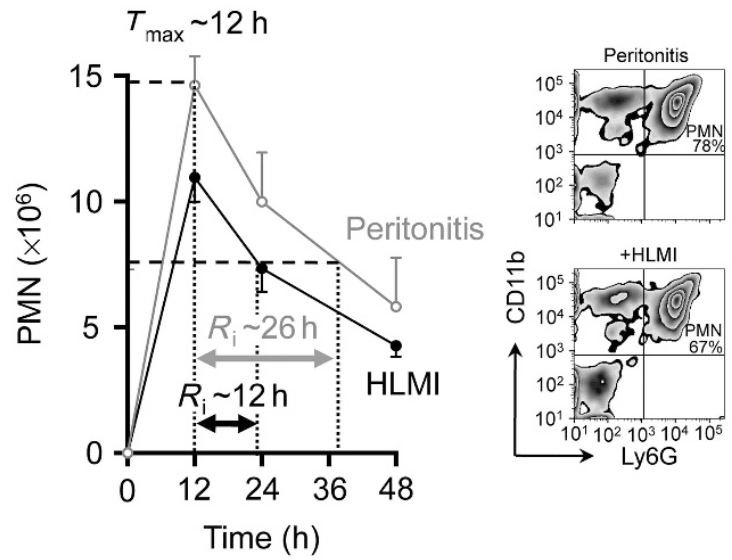

\begin{tabular}{|c|c|c|c|}
\hline $\begin{array}{l}\text { Resolution } \\
\text { Indices }\end{array}$ & $\begin{array}{c}\text { Peritonitis } \\
+ \text { vehicle }\end{array}$ & $\begin{array}{c}\text { Peritonitis } \\
+\mathrm{HLMI}\end{array}$ & $\%$ reduction \\
\hline$\Psi_{\max } ;\left(\times 10^{6}\right)$ & $14.6 \pm 1.2$ & $11.0 \pm 1.0$ & $23.1 \pm 8.9 \%$ \\
\hline$T_{\max } ;(\mathrm{h})$ & 12.0 & 12.0 & - \\
\hline$T_{50} ;(\mathrm{h})$ & $\sim 38.0$ & $\sim 24.0$ & $\sim 37 \%$ \\
\hline$R_{\mathrm{i}} ;(\mathrm{h})$ & $\sim 26.0$ & $\sim 12.0$ & $\sim 54 \%$ \\
\hline
\end{tabular}

C

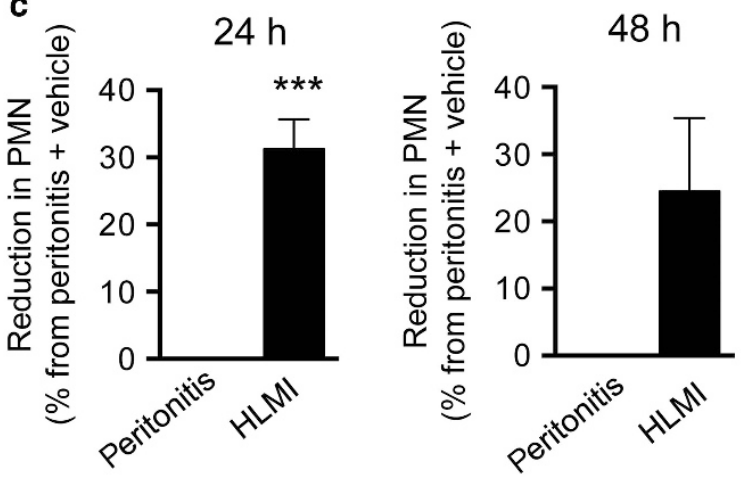

Figure 1 Human milk lipid mediator isolates (HLMIs) shorten resolution of acute inflammation. (a) Exudate polymorphonuclear cell (PMN) numbers in mice administered vehicle or HLMls (in $200 \mu \mathrm{l}$ saline, intraperitoneally) immediately before self-limited inflammatory challenge (zymosan; $1 \mathrm{mg}$, intraperitoneally). Inset, representative flow cytometry zebra plot; PMN identified as CD11b ${ }^{+} \mathrm{Ly}_{6 \mathrm{G}}{ }^{+}$events. (b) Resolution indices were determined: $\Psi_{\max }$ (maximal PMN counts), $T_{\max }$ (the time interval when PMN reach maximum), $T_{50}$ (the time interval corresponding to $50 \%$ PMN reduction, or $\Psi_{50}$ ) and $R_{\mathrm{i}}$ (resolution interval, the interval between $T_{\max }$ and $T_{50}$; see Methods for details). (c) Reduction in PMN numbers at 24 and $48 \mathrm{~h}$ from peritonitis plus vehicle mice. Results are mean \pm s.e.m.; ${ }^{* \star *} P<0.001$ vs. vehicle; $n=4$ mice per treatment at each time interval.

demonstrate that human milk possesses proresolving properties contained in the HLMIs.

\section{Human milk LM-SPM signature profile: LM metabololipidomics}

Because isolates from human milk accelerate resolution (Figure 1), we next sought to investigate the LM profile of human milk. Using liquid-chromatography tandem-mass spectrometry (LC-MS-MS)-based LM metabololipidomics (see Methods for details), we identified a profile signature 


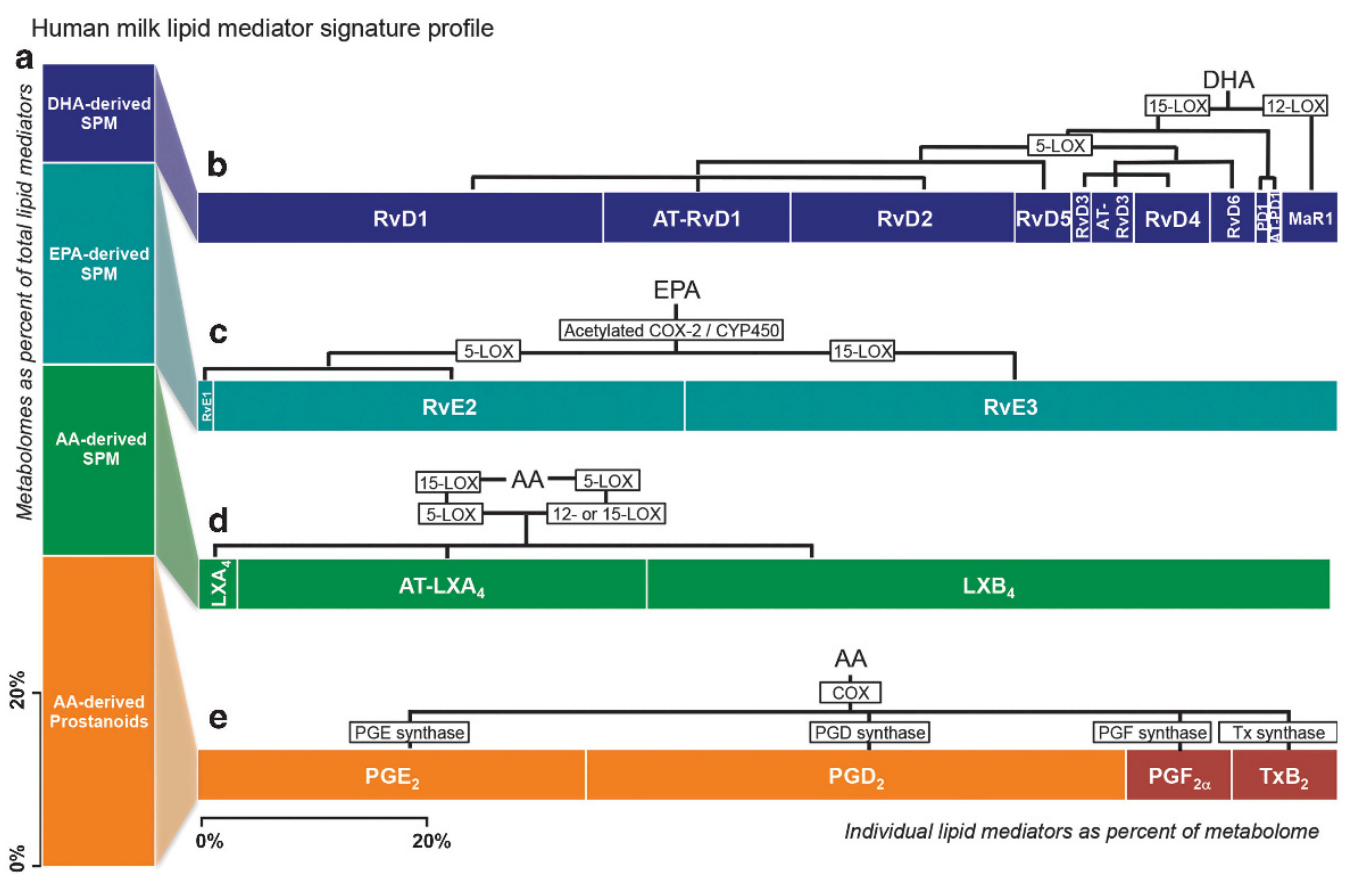

Figure 2 Signature lipid mediator-specialized proresolving mediator (LM-SPM) profile of human milk. LMs obtained from human milk (4-8 weeks postpartum) were identified by liquid-chromatography tandem-mass spectrometry (LC-MS-MS)-based LM metabololipidomics (see Methods for details). (a) Percentage of docosahexaenoic acid (DHA)-derived SPM, eicosapentaenoic acid (EPA)-derived SPM, arachidonic acid (AA)-derived SPM, and AAderived prostanoids in human milk from healthy volunteers. (b-e) Contribution of individual LMs and SPMs within each metabolome. Biosynthetic pathways are indicated above each major essential fatty acid (EFA) metabolome (DHA, EPA, and AA). Vertical bars represent \% of total LMs (ng) from $n=4$ healthy human milk donors. COX-2, cyclooxygenase-2; LOX, lipoxygenase; LX, lipoxin; MaR, maresin; PD, protectin; PGE ${ }_{2}$, prostaglandin $\mathrm{E}_{2}$; $\mathrm{PGF}_{2 \alpha}$, prostaglandin $\mathrm{F}_{2 \alpha} ; \mathrm{PGD}_{2}$, prostaglandin $\mathrm{D}_{2} ; \mathrm{RvD}$, D-series Rv; RvE, E-series resolvin; $\mathrm{TxB}_{2}$, thromboxane $\mathrm{B}_{2}$.

of LMs consisting of 20 bioactive LMs (Figure 2, Table 1, and Supplementary Figure 1 online) from both lipoxygenase and cyclooxygenase pathways, including Rvs, PDs, MaRs, LXs, and prostanoids (Figure 2, Table 1, Supplementary Figure 1, and Supplementary Table 1). Each LM was identified by matching LC retention time and at least six diagnostic ions, and quantification was achieved using multiple reaction monitoring in accordance with published criteria, ${ }^{3}$ and as illustrated with representative results obtained for all identified LMs (Supplementary Figure 1b).

LM quantification, using multiple reaction monitoring, demonstrated that SPMs in healthy mature human milk (4-8 weeks postpartum) include AT-RvD1 $\left(67.4 \pm 11.7 \mathrm{pg} \mathrm{ml}^{-1}\right)$, RvD2 $\left(82.4 \pm 28.0 \mathrm{pg} \mathrm{ml}^{-1}\right)$, RvD3 $\left(7.2 \pm 2.7 \mathrm{pg} \mathrm{ml}^{-1}\right)$, ATRvD3 $\left(15.0 \pm 2.9 \mathrm{pg} \mathrm{ml}^{-1}\right)$, RvD4 $\left(27.4 \pm 7.5 \mathrm{pg} \mathrm{ml}^{-1}\right)$, RvD5 $\left(19.9 \pm 8.9 \mathrm{pg} \mathrm{ml}^{-1}\right), \quad$ RvD6 $\quad\left(6.7 \pm 2.4 \mathrm{pg} \mathrm{ml}^{-1}\right), \quad$ PD1 $\left(4.3 \pm 2.3 \mathrm{pg} \mathrm{ml}^{-1}\right)$, AT-PD1 $\left(3.8 \pm 0.9 \mathrm{pg} \mathrm{ml}^{-1}\right)$, and MaR1 $\left(20.8 \pm 6.3 \mathrm{pg} \mathrm{ml}^{-1}\right)$ from the DHA metabolome, RvE2 $\left(321.2 \pm 129.2 \mathrm{pg} \mathrm{ml}^{-1}\right)$ and $\operatorname{RvE} 3\left(444.9 \pm 179.8 \mathrm{pg} \mathrm{ml}^{-1}\right)$ from the EPA metabolome, and AT-LXA $\left(370.0 \pm 176.6 \mathrm{pg} \mathrm{ml}^{-1}\right)$ and $\mathrm{LXB}_{4}\left(267.1 \pm 93.9 \mathrm{pg} \mathrm{ml}^{-1}\right)$ from the AA metabolome (Table 1). These are in addition to RvD1 $\left(147.0 \pm 47.2 \mathrm{pg} \mathrm{ml}^{-1}\right)$, $\operatorname{RvE} 1\left(8.8 \pm 3.6 \mathrm{pg} \mathrm{ml}^{-1}\right)$, and $\mathrm{LXA}_{4}\left(25.7 \pm 8.6 \mathrm{pg} \mathrm{ml}^{-1}\right)$. These confirm the identification of RvD1, RvE1, and $\mathrm{LXA}_{4}$ in human milk, at values consistent with those recently reported. ${ }^{6}$ From the cyclooxygenase pathways, we also identified prostaglandin $\mathrm{E}_{2}\left(\mathrm{PGE}_{2}\right)\left(409.7 \pm 146.6 \mathrm{pg} \mathrm{ml}^{-1}\right)$, prostaglandin $\mathrm{D}_{2} \quad\left(\mathrm{PGD}_{2}\right) \quad\left(568.3 \pm 188.9 \mathrm{pg} \mathrm{ml}^{-1}\right), \quad$ prostaglandin $\mathrm{F}_{2 \alpha}$ $\left(\mathrm{PGF}_{2 \alpha}\right)\left(111.1 \pm 36.2 \mathrm{pg} \mathrm{ml}^{-1}\right)$, and thromboxane $\mathrm{B}_{2}\left(\mathrm{TxB}_{2}\right)$ $\left(111.8 \pm 44.4 \mathrm{pg} \mathrm{ml}^{-1}\right)$ in these samples in accordance with published findings. ${ }^{15}$ These results demonstrate that human milk contains SPMs at biologically relevant concentrations.

Next, we determined the contribution of each of the major bioactive metabolomes (DHA, EPA, and $\mathrm{AA}$ ) as well as individual mediators within each metabolome to the human milk LM signature profile (Figure 2). LM metabololipidomics of human milk AA, EPA, and DHA identified bioactive metabolome demonstrated that SPMs represented $\sim 61.6 \%$ of the human milk LM profile (Figure 2), consisting of DHAderived Rvs, PDs, and MaRs (13.1\%), AA-derived LXs (23.5\%), and EPA-derived Rvs (24.9\%; Figure 2). AA-derived prostanoids amounted to $\sim 38.4 \%$ of the LMs identified (Figure 2), consisting primarily of $\mathrm{PGE}_{2}$ and $\mathrm{PGD}_{2}(\sim 81.5 \%$ of total prostanoids) that are key in LM mediator class switching and initiation of resolution. ${ }^{16}$ Of primary proinflammatory $\mathrm{LM}, \mathrm{PGF}_{2 \alpha}$ and $\mathrm{TxB}_{2}$, an inactive further metabolite of $\mathrm{TxA}_{2}{ }^{17}$ combined amounted to $<10 \%$ of total milk LMs (Figure 2). $\mathrm{LTB}_{4}$ is a potent proinflammatory neutrophil chemoattractant ${ }^{18}$ and was not identified in appreciable amounts in these milk samples (Table 1). This approach permitted us to assess the potential effector functions that human milk LM-SPM may endow locally within the mammary 
Table 1 Bioactive LM profile of human milk (4-8 weeks postpartum)

\begin{tabular}{|c|c|c|c|c|}
\hline & \multirow[b]{2}{*}{ Q1 } & \multirow[b]{2}{*}{ Q3 } & \multicolumn{2}{|c|}{$\begin{array}{l}\text { Healthy human milk, } \\
\text { 4-8 weeks postpartum }\end{array}$} \\
\hline & & & $\begin{array}{l}\text { Lipid mediator } \\
\text { levels }\left(\mathrm{pg} \mathrm{ml}^{-1}\right)\end{array}$ & рм \\
\hline \multicolumn{5}{|c|}{ DHA bioactive SPM metabolome } \\
\hline RvD1 & 375 & 215 & $147 \pm 47.2$ & 391 \\
\hline AT-RvD1 & 375 & 215 & $67.4 \pm 11.7$ & 180 \\
\hline RvD2 & 375 & 215 & $82.4 \pm 28.0$ & 219 \\
\hline RvD3 & 375 & 147 & $7.2 \pm 2.7$ & 19.1 \\
\hline AT-RvD3 & 375 & 147 & $15.0 \pm 2.9$ & 39.9 \\
\hline RvD4 & 375 & 101 & $27.4 \pm 7.5$ & 72.9 \\
\hline RvD5 & 359 & 199 & $19.9 \pm 8.9$ & 52.9 \\
\hline RvD6 & 359 & 101 & $6.7 \pm 2.4$ & 17.8 \\
\hline PD1 & 359 & 153 & $4.3 \pm 2.3$ & 11.9 \\
\hline AT-PD1 & 359 & 153 & $3.8 \pm 0.9$ & 10.6 \\
\hline MaR1 & 359 & 221 & $20.8 \pm 6.3$ & 57.8 \\
\hline \multicolumn{5}{|c|}{ AA bioactive $L M$ metabolome } \\
\hline $\mathrm{LXA}_{4}$ & 351 & 115 & $25.7 \pm 8.6$ & 72.9 \\
\hline AT-LXA 4 & 351 & 115 & $370.0 \pm 176.6$ & 1,260 \\
\hline $\mathrm{LXB}_{4}$ & 351 & 115 & $267.1 \pm 93.9$ & 759 \\
\hline AT-LXB ${ }_{4}$ & 351 & 115 & - & - \\
\hline $\mathrm{LTB}_{4}$ & 335 & 195 & - & - \\
\hline $\mathrm{PGE}_{2}$ & 351 & 189 & $409.7 \pm 146.6$ & 1,160 \\
\hline$P G D_{2}$ & 351 & 189 & $568.3 \pm 188.9$ & 1,610 \\
\hline $\mathrm{PGF}_{2 \alpha}$ & 353 & 193 & $111.1 \pm 36.2$ & 314 \\
\hline $\mathrm{TxB}_{2}$ & 369 & 169 & $111.8 \pm 44.4$ & 302 \\
\hline
\end{tabular}

EPA bioactive SPM metabolome

\begin{tabular}{lrrrr} 
RVE1 & 349 & 195 & $8.8 \pm 3.6$ & 25.1 \\
RvE2 & 333 & 253 & $321.2 \pm 129.2$ & 962 \\
RvE3 & 333 & 201 & $444.9 \pm 179.8$ & 1,330 \\
\hline
\end{tabular}

Abbreviations: AA, arachidonic acid; DHA, docosahexaenoic acid; EPA, eicosapentaenoic acid; LC-MS-MS, liquid-chromatography tandem-mass spectrometry; $L M$, lipid mediator; $\mathrm{LTB}_{4}$, leukotriene $\mathrm{B}_{4}$; $\mathrm{LXB}_{4}$, lipoxin $\mathrm{B}_{4} ; \mathrm{MaR}$, maresin; $P D$, protectin; $\mathrm{PGD}_{2}$, prostaglandin $\mathrm{D}_{2} ; \mathrm{PGE}_{2}$, prostaglandin $\mathrm{E}_{2} ; \mathrm{PGF}_{2 \alpha}$, prostaglandin $\mathrm{F}_{2 \alpha}$; RvD, D-series resolvin; RvE, E-series resolvin; SPM, specialized proresolving mediator; $\mathrm{TxB}_{2}$, thromboxane $\mathrm{B}_{2}$

Quantification of bioactive LMs in human milk (4-8 weeks postpartum), as assessed by LC-MS-MS based LM metabololipidomics. Results are expressed as $\mathrm{pg} \mathrm{ml}^{-1}$ human milk. Q1: M-H (parent ion) and Q3 (daughter ion): diagnostic ion in the MSMS. Detection limit was $\sim 0.1 \mathrm{pg}$; - : denotes below limits. Results are mean \pm s.e.m. from four donors. Complete LC-MS-MS, retention times, and MS-MS spectra for each eicosanoid and SPMs listed here are presented in Supplementary Figure 1. gland or on the infant during maternal-infant transfer. Taken together, these results demonstrate that human milk contains a proresolving LM-SPM signature profile, comprised predominantly of LMs and SPMs with proresolving properties at concentrations commensurate with their known bioactions. ${ }^{1,17,18}$

\section{Human milk LM-SPM profile is altered in mastitis}

SPMs are endogenous chemical signals that actively stimulate resolution of inflammation; ${ }^{1}$ therefore, we next sought to investigate the LM profiles of human milk from inflamed mammary glands (mastitis) and compare it with milk from healthy subjects (Figure 3). Differences in LM-SPM profiles obtained with human milk from healthy individual donors (1-6 months postpartum) and donors with mastitis (1-4 months postpartum) were assessed using principal component analysis. The two principal components, calculated using the data matrix, showed clear separation between the healthy milk cluster and mastitis milk cluster on the score plot (Figure 3a). The healthy milk cluster was characterized by higher levels of SPMs, including RvD1, RvD2, RvD3, MaR1, PD1, RvE2, and $\mathrm{LXA}_{4}$ and $\mathrm{LXB}_{4}$ as demonstrated in the loading plot (Figure $3 \mathbf{b}$ ). Conversely, principal component analysis of the LC-MS-MS results demonstrated that the mastitis milk cluster was associated with higher levels of RvE1, $\mathrm{LTB}_{4}, \mathrm{PGD}_{2}$, $\mathrm{PGF}_{2 \alpha}$, and $\mathrm{TxB}_{2}$. These findings indicate that the human milk LM profile is altered in mastitis, with elevated proinflammatory LMs and reduced SPMs.

As mastitis milk had an altered LM-SPM profile, we next investigated the ability of HLMIs from mastitis milk (referred to as $\mathrm{HLMI}_{\text {mast }}$ ) to accelerate resolution of acute inflammation. HLMI from mastitis milk was obtained as described above for HLMIs from healthy milk (see Methods for details). Administration of $\mathrm{HLMI}_{\text {mast }}$ immediately before challenge $(1 \mathrm{mg}$ zymosan per mouse) did not limit neutrophil numbers at $T_{\max }$ $(12.3 \pm 0.8$ vs. $11.5 \pm 0.9$ cells per exudate compared with peritonitis plus vehicle), and only slightly shortened the $R_{\mathrm{i}}$ by $16 \%$, or from $19 \mathrm{~h}$ observed in peritonitis plus vehicle to $16 \mathrm{~h}$ (Figure $3 \mathbf{c}$ and $\mathbf{d}$ ). Taken together, these findings indicate that mastitis milk has altered LM-SPM signature profile and reduced ability to accelerate resolution in vivo.

\section{RvD2 and MaR1 potently accelerate resolution of acute inflammation}

Because DHA is recognized to be critical for neonatal development ${ }^{10}$ and RvD2 was one of the more abundant DHA-derived SPMs identified in human milk (Figure 2 and Table 1), we sought to assess its potential contribution to the regulation of leukocyte trafficking and the $R_{\mathrm{i}}$. Mice were administered RvD2 (50 ng per mouse, i.e., $2 \mathrm{\mu g} \mathrm{kg}^{-1}$; intraperitoneally) before initiation of a self-limited inflammatory challenge and resolution parameters quantified (Figure 4). RvD2 gave $\sim 40 \%$ reduction in $\Psi_{\max }$ $\left(10.0 \pm 0.8 \times 10^{6}\right.$ vs. $17.0 \pm 2.4 \times 10^{6}$ cells per exudate $)$ compared with peritonitis plus vehicle mice and shortened the $R_{\mathrm{i}}$ by $74 \%$, or from 25 to $6.5 \mathrm{~h}$ (Figure 4). DHA also serves as a substrate for MaRs, ${ }^{1}$ and as MaR1 was identified in human milk at bioactive concentrations (Figure 2 and Table 1) we compared its actions 
a

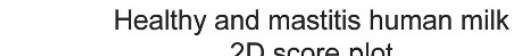
2D score plot

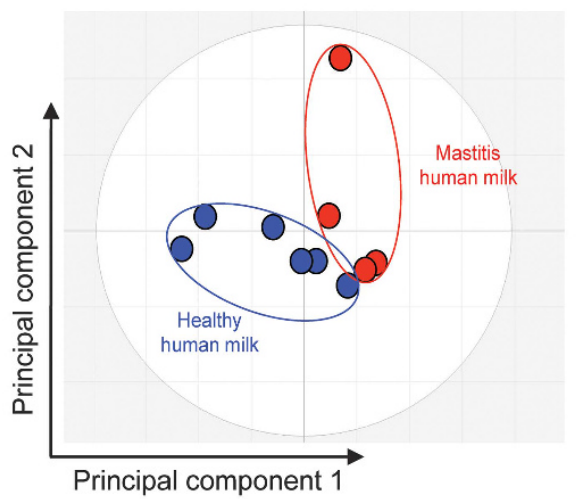

C

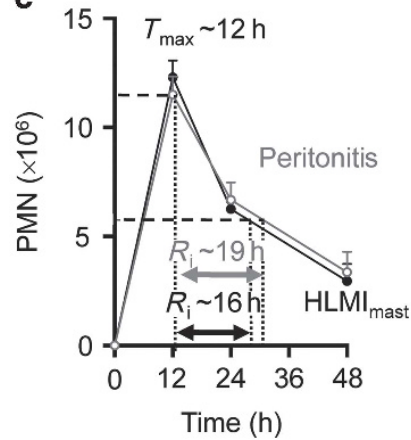

b Healthy and mastitis human milk $2 \mathrm{D}$ loading plot

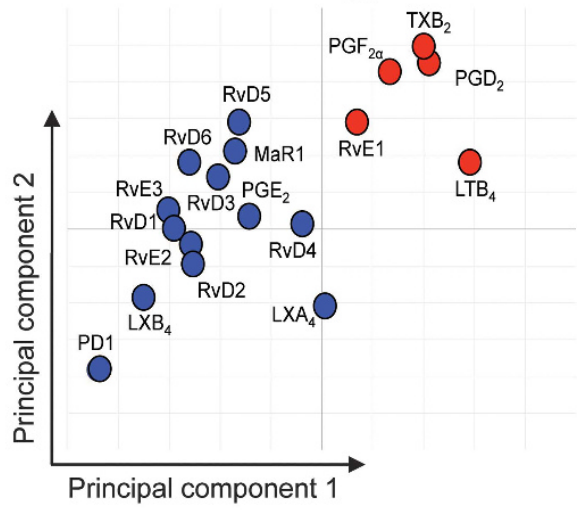

d

\begin{tabular}{lccc}
$\begin{array}{l}\text { Resolution } \\
\text { Indices }\end{array}$ & $\begin{array}{l}\text { Peritonitis Peritonitis } \\
+ \text { vehicle }+\mathrm{HLMI}_{\text {mast }}\end{array}$ & $\%$ change \\
\hline$\Psi_{\text {max }} ;\left(\times 10^{6}\right)$ & $11.5 \pm 0.9$ & $12.3 \pm 0.8$ & $+7.1 \pm 2.1 \%$ \\
$T_{\text {max }} ;(\mathrm{h})$ & 12.0 & 12.0 & - \\
$T_{50} ;(\mathrm{h})$ & $\sim 31.0$ & $\sim 28.0$ & $-10 \%$ \\
$R_{\mathrm{i}} ;(\mathrm{h})$ & $\sim 19.0$ & $\sim 16.0$ & $-16 \%$
\end{tabular}

Figure 3 Mastitis human milk has altered lipid mediator-specialized proresolving mediator (LM-SPM) profiles and reduced ability to accelerate resolution. LMs obtained from healthy and mastitis-affected human milk (1-6 months postpartum) were identified by liquid-chromatography tandemmass spectrometry (LC-MS-MS) metabololipidomics (see Methods for details). (a) Two-dimensional (2D) score plot of human milk from healthy donors $(n=7)$ compared with mastitis donors $(n=4)$. (b) 2D loading plot. (c) Exudate polymorphonuclear cell (PMN) numbers in mice administered vehicle or HLMI from mastitis milk ( $\mathrm{HLMI}_{\text {mast; }}$ in $200 \mu \mathrm{l}$ saline, intraperitoneally) immediately before self-limited inflammatory challenge (zymosan; 1 mg, intraperitoneally). Inset, representative flow cytometry zebra plot; PMN identified as CD11 b ${ }^{+}$Ly6G ${ }^{+}$events. (d) Resolution indices were calculated as in Figure 1 (see Methods for details). Results are mean \pm s.e.m.; $n=3$ mice per treatment at each time interval. $L_{T B}$, leukotriene $B_{4} ; L_{X}$, lipoxin; MaR1, maresin 1; PD1, protectin D1; $\mathrm{PGE}_{2}$, prostaglandin $\mathrm{E}_{2} ; \mathrm{PGF}_{2 \alpha}$, prostaglandin $\mathrm{F}_{2 \alpha} ; \mathrm{PGD}_{2}$, prostaglandin $\mathrm{D}_{2} ; \mathrm{RvD}$, D-series Rv; TxB $\mathrm{B}_{2}$, thromboxane $\mathrm{B}_{2}$.

on regulating leukocyte trafficking to RvD2. MaR1 (50 ng per mouse, intraperitoneally) gave a maximal PMN number of $9.9 \pm 1.3 \times 10^{6}$ cells per exudate and shortened the $R_{\mathrm{i}}$ to $6 \mathrm{~h}$, or by $76 \%$ (Figure 4). We also assessed the ability of RvD2 and MaR1 to accelerate resolution of established peritonitis (Supplementary Figure 2a, b). RvD2 and MaR1 (50 ng per mouse) administered $12 \mathrm{~h}$ after zymosan challenge (1 mg per mouse) each accelerated resolution, reducing neutrophil numbers and shortening the $R_{\mathrm{i}}$ by $33 \%$ and $40 \%$, respectively (Supplementary Figure 2a, b). Thus, both RvD2 and MaR1, at physiologic range, that is, nanograms per mouse, regulate neutrophil trafficking and shorten the $R_{\mathrm{i}}$.

\section{HLMIs and MaR1 stimulate resolution of infectious peritonitis}

Given these in vivo findings and as HLMIs contain SPMs that enhance host-directed responses to infection, such as RvD1, RvD5, and RvD2, ${ }^{19,20}$ we next investigated whether HLMIs enhanced resolution of infectious peritonitis (Supplementary Figure $3 \mathbf{a}, \mathbf{b})$. Mice were inoculated with a resolving dose of Escherichia coli $\left(10^{5}\right.$ colony-forming unit and administered vehicle or HLMIs (intraperitoneally) $12 \mathrm{~h}$ later. HLMIs gave reduced PMN numbers at $24 \mathrm{~h}$ by $33 \%(9.8 \pm 1.1$ vs. $14.6 \pm 1.8$ cells per exudate compared with peritonitis plus vehicle; Supplementary Figure 3a) and enhanced leukocyte uptake of E. coli (Supplementary Figure 3b). As MaR1 potently accelerated resolution of sterile inflammation and is present in human milk, we assessed its ability to enhance resolution of infection (Supplementary Figure 3c, d). We found that MaR1 (50 ng per mouse) reduced PMN numbers at $24 \mathrm{~h}$ by $40 \%$ (Supplementary Figure 3c) and enhanced leukocyte uptake of E. coli (Supplementary Figure 3d). Similar results were obtained with $\operatorname{RvD2}$ ( $n=2$, data not shown) used for direct comparison. $^{20}$ Taken together, these results demonstrate that HLMIs and MaR1 accelerate resolution of infection, limiting neutrophil numbers and enhancing in vivo bacterial clearance.

\section{HLMIs enhance human macrophage phagocytosis}

Given the key actions of SPMs in resolution are enhancing macrophage clearance of apoptotic cells and debris, ${ }^{1}$ we next questioned whether HLMIs have direct impact on phagocytosis with isolated human cells. Incubation of human macrophages 

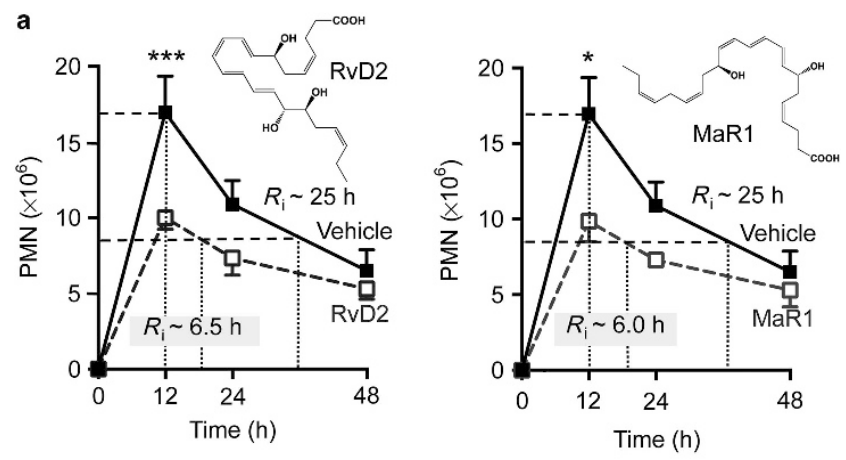

\begin{tabular}{lccc}
$\begin{array}{c}\text { besolution } \\
\text { Indices }\end{array}$ & $\begin{array}{c}\text { Peritonitis } \\
\text { vehicle }\end{array}$ & $\begin{array}{c}\text { Peritonitis }+ \\
\text { RvD2 }\end{array}$ & $\begin{array}{c}\text { Peritonitis }+ \\
\text { MaR1 }\end{array}$ \\
\hline$\Psi_{\text {max }} ;\left(\times 10^{6}\right)$ & $17.0 \pm 2.4$ & $10.0 \pm 0.8$ & $9.9 \pm 1.3$ \\
$T_{\text {max }} ;(h)$ & 12.0 & 12.0 & 12.0 \\
$T_{50} ;(h)$ & $\sim 37.0$ & $\sim 18.5$ & $\sim 18.0$ \\
$R_{i} ;(h)$ & $\sim 25.0$ & $\sim 6.5$ & $\sim 6.0$ \\
Shortening of $\mathbf{R}_{\boldsymbol{i}}$ & - & $74 \%$ & $76 \%$ \\
\hline
\end{tabular}

Figure 4 Resolvin D2 (RvD2) and maresin 1 (MaR1) accelerate resolution of inflammation. (a) Exudate polymorphonuclear cell (PMN) numbers in mice administered vehicle, RvD2 (left) or MaR1 (right; $50 \mathrm{ng}$ each per mouse, intraperitoneally) before injection of zymosan (1 mg per mouse, intraperitoneal). Inset: molecular structure for RvD2 (left) and MaR1 (right). (b) Resolution indices were calculated as in Figure 1 (see Methods for details). Results are mean \pm s.e.m.; ${ }^{*} P<0.05$ and

${ }^{* * *} P<0.001$ vs. vehicle; $n=6$ mice (vehicle and RvD2) or $n=3$ mice (MaR1) at each time interval.

with HLMIs gave an enhanced efferocytosis (i.e., phagocytosis of fluorescently labeled apoptotic neutrophils) compared with vehicle-treated macrophages (Figure 5a). To provide evidence whether the LMs found in HLMIs are responsible for the potent bioactions, we depleted LMs from human milk using activated charcoal $^{21}$ (referred to here as $\mathrm{HLMI}_{\mathrm{AC}}$ ) and compared its actions with that of HLMIs. Charcoal treatment depleted more than $\sim 97 \%$ of the bioactive LM content of human milk (DHAderived SPM: 23.3 vs. 0.1 pg per $20 \mu$ lisolate; AA-derived SPM: 35.2 vs. $1.3 \mathrm{pg}$ per $20 \mu \mathrm{l}$ isolate; EPA-derived SPM: 78.5 vs. $3.3 \mathrm{pg}$ per $20 \mu \mathrm{l}$ isolate; AA-derived prostanoids: 155.8 vs. $10.6 \mathrm{pg}$ per $20 \mu \mathrm{l}$ isolate) and significantly reduced the ability of the HLMIs to stimulate macrophage efferocytosis by 80-95\% (Figure 5a). Thus, HLMIs possess bioactive SPMs that stimulate key resolution programs in human macrophages, namely efferocytosis.

Based on these and the in vivo findings, and as SPM, including RvD1, RvD2, and RvD5, directly enhance human phagocyte containment of E. coli, ${ }^{19,20}$ we next questioned whether HLMIs have direct impact on bacterial containment with isolated human cells. HLMIs increased human macrophage phagocytosis of fluorescent E. coli by $\sim 35-55 \%$ compared with vehicletreated macrophages (Figure $\mathbf{5 b}$ ). The ability of HLMIs to enhance macrophage containment of E. coli was significantly reduced after LM depletion with activated charcoal (Figure 5b). Taken together, these results demonstrate that HLMI possesses bioactive LMs/SPMs that enhance bacterial containment with isolated human macrophages.
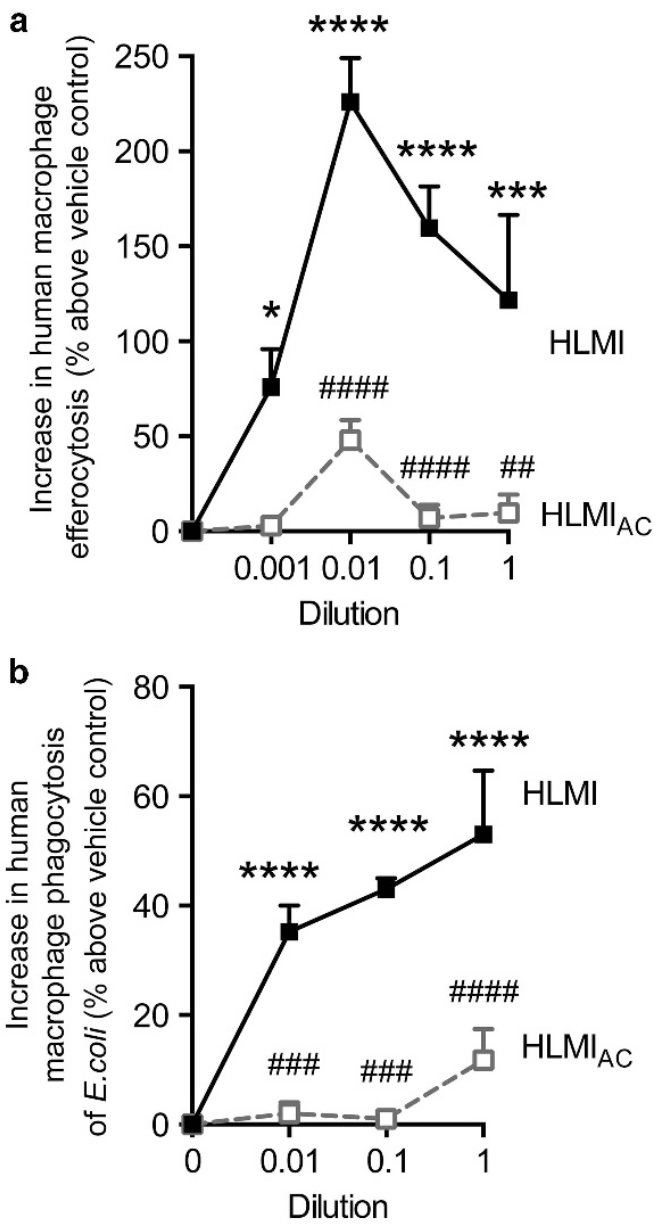

Figure 5 Human milk lipid mediator isolates (HLMls) enhance human macrophage phagocytosis. Enhanced phagocytosis of (a) apoptotic polymorphonuclear cell (PMN) and (b) Escherichia coli (E. colli) with human macrophages expressed as increase in phagocytosis above vehicle. Macrophages $\left(5 \times 10^{4}\right.$ cells per well) were incubated with indicated concentration of HLMls or HLMI depleted of LMs by activated charcoal $\left(\mathrm{HLMI}_{\mathrm{AC}}\right)(1=$ highest concentration, $0.1=1: 10$ dilution, $0.01=1: 100$ dilution, $0.001=1: 1000$ dilution; $\mathrm{pH} 7.45$ at $37^{\circ} \mathrm{C}$ for $15 \mathrm{~min}$ ). Subsequently, fluorescently labeled (a) apoptotic PMN (3:1

$\mathrm{PMN}$ :macrophage) or (b) E. coli (50:1 E. coli:macrophages) were added $\left(45 \mathrm{~min}\right.$ at $37^{\circ} \mathrm{C}$ ). Nonphagocytosed apoptotic PMN or E. coli were washed off, extracellular fluorescence quenched, and phagocytosis determined using a fluorescence plate reader. Results are mean \pm s.e.m.; ${ }^{*} P<0.05,{ }^{* * *} P<0.001,{ }^{* * *} P<0.0001$ vs. vehicle; ${ }^{\# \#} P<0.01$,

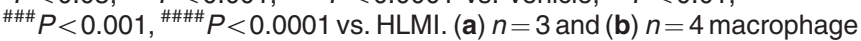
donors.

\section{DISCUSSION}

In the present study, we report the human milk LM-SPM signature profile that signals resolution of inflammation and bacterial clearance. Using LC-MS-MS-based LM metabololipidomics, we identified Rvs, PDs, MaRs, and LXs at bioactive concentrations in healthy human milk. For comparison, in mastitis, milk LM-SPM levels were altered showing elevated proinflammatory LMs and lower levels of SPMs. RvD2 and MaR1 were identified in human milk, and each individually accelerated resolution of inflammation, shortening the $R_{\mathrm{i}}$ from 26 to $12 \mathrm{~h}$. Also, HLMIs had infection-resolving actions in vivo, 
enhanced efferocytosis, and phagocytosis of E. coli with isolated human macrophages.

Human milk is a dynamic biologically active fluid that in addition to delivering essential nutrients provides passive protection for the immature mucosal immune system. ${ }^{10}$ Owing to the immaturity of the intestinal immune system in newborns, they have enhanced susceptibility to excessive inflammation and infection. ${ }^{12}$ Recently, chemical signals that actively stimulate resolution of inflammation and infection ${ }^{1}$ were identified in human milk. ${ }^{6}$ Of note, SPMs, such as Rvs, PDs, MaRs, and LXs, are endogenous LMs found in many tissues that actively counterregulate proinflammatory signals, including nuclear factor- $\kappa \mathrm{B},{ }^{9}$ cytokines, and leukotrienes. ${ }^{1}$ They exert their potent actions via activating specific G-protein-coupled receptors in cell-specific and tissue-dependent manner. Several SPM receptors are identified, for example, RvE1 specifically binds both ChemR23 and BLT1, and LXA ${ }_{4}$ and RvD1 bind and activate the LX $\mathrm{A}_{4}$ receptor ALX and human GPR32, which also binds RvD3 and RvD5 (reviewed in Serhan ${ }^{1}$ ). RvD2 was recently found to exert its tissue-protective actions via GPR18. ${ }^{22}$ Along these lines, enterocytes express $\mathrm{ALX}^{23}$ and $\mathrm{LXA}_{4}$ stable analogs inhibit bacterial-induced interleukin-8 secretion by intestinal epithelial cells. ${ }^{24}$ Enterocytes also express ChemR23, where RvE1 induces intestinal alkaline phosphatase expression and enzyme activity that attenuates lipopolysaccharide-induced nuclear factor- $\mathrm{\kappa B}$ signaling. ${ }^{25}$ Hence, together with our present results SPMs in human milk may be relevant for infant mucosal responses. Given their presence at bioactive levels in human milk (рм to nм) and their ability to engage G-protein-coupled receptors, they may activate specific and potentially additive responses in the newborn gut mucosa; such actions remain of interest.

Proresolution is a distinct process from anti-inflammation, where agonists of resolution, such as SPM, augment nonphlogistic clearance from sites of inflammation and infection, augmenting host-directed defenses including microbial containment. ${ }^{19,26}$ In the present report, we found that human milk isolates containing SPMs accelerate resolution of acute inflammation and infection in vivo and with isolated human leukocytes. Mastitis milk gave altered SPM levels and reduced ability to accelerate resolution of acute inflammation. The higher RvE1 levels in mastitis milk may reflect an increased cytochrome $P 450$ in the mastitis microenvironment, for example, cytochrome $P 450$ can produce the RvE1 precursor 18-HEPE (hydroxyicosapentaenoic acid) from EPA, which in turn is converted to RvE1 by human PMN (reviewed in Serhan ${ }^{1}$ ), which are known to be abundant in mastitis-affected milk. ${ }^{27}$ In addition to the known beneficial properties of human milk, our current results extend its protective roles to now include proresolving properties, namely accelerating resolution of acute inflammation and infection, as well as stimulating macrophage phagocytic functions with the LC-MS-MS-based identification of human milk SPMs.

Resolution of acute inflammation can be quantitated using defined resolution indices introduced by this laboratory. ${ }^{13,14}$ These permit direct assessment of proresolving properties of
Table 2 Comparison of SPM impact on the $R_{\mathrm{i}}$ in mouse peritonitis $^{a}$

\begin{tabular}{lccc}
\hline Agonist & Dose & $\begin{array}{c}\text { Shortening of } \\
\boldsymbol{R}_{\mathrm{i}}(\%)\end{array}$ & $\begin{array}{c}\text { Reduction } \\
\text { of }\end{array}$ \\
\hline RvD1 & 50 ng per mouse max $^{19}$ & 40 & 40 \\
RvD2 & 50 ng per mouse & 74 & 41 \\
RvD3 & 50 ng per mouse & 92 & 47 \\
MaR1 & 50 ng per mouse & 76 & 58 \\
\hline
\end{tabular}

Abbreviations: LM, lipid mediator; MaR, maresin; $R_{\mathrm{i}}$, resolution interval; RvD, D-series resolvin; SPM, specialized proresolving mediator.

aThe impact of SPMs administered intraperitoneally (at initiation of inflammation) on resolution interval in murine self-limited peritonitis initiated by intraperitoneal injection of $1 \mathrm{mg}$ zymosan or E. coli $\left(10^{5}\right.$ colony-forming units, c.f.u). For direct comparison, see Chiang et al. ${ }^{19}$ and Arnardottir et al. ${ }^{41}$

endogenous mediators (Table 2 and Supplementary Table 2). For example, RvD1 and RvD3 (50 ng per mouse, i.e., $2 \mu \mathrm{gg}^{-1}$, each) shorten $R_{\mathrm{i}}$ in murine peritonitis (Table 2). Also, RvD1, $\mathrm{PD} 1$, and $\mathrm{AT}-\mathrm{LXA}_{4}$ at $300 \mathrm{ng}$ per mouse (i.e., $12 \mu \mathrm{g} \mathrm{kg}^{-1}$ ) each reduce the $R_{\mathrm{i}}$, whereas RvE1 accelerates the onset $\left(T_{\max }\right)$ of resolution (Supplementary Table 2). In these experiments, RvD2 and MaR1 accelerate resolution of acute inflammation, reducing the magnitude of PMN infiltration $\left(\Psi_{\max }\right)$ and shortening $R_{\mathrm{i}}$. Of note, RvD2 and MaR1 each limit intestinal inflammation and tissue damage in experimental colitis. ${ }^{9,28}$ Of interest, oral administration of RvD1 shortens the $R_{\mathrm{i}}{ }^{29}$ Hence, taken together with our finding that SPMs, including $\mathrm{RvD} 2$ and MaR1, are present in human milk at biologically relevant levels, SPMs and their pathways may have implications in the regulation of acute inflammation and resolution in maternal-infant transferred protection.

Emerging evidence indicates that breastfeeding is correlated with lower prevalence of inflammatory conditions in early life (e.g., necrotizing enterocolitis) and later life (e.g., obesity, diabetes, and cardiovascular disease). ${ }^{30}$ Human milk contains high levels of EFA, such as DHA, which are derived from maternal dietary and endogenous pools (e.g., adipose tissue). ${ }^{31}$ Increased maternal intake of $n-3$ EFA during gestation and lactations has been associated with beneficial outcome for infants. ${ }^{10}$ Also, DHA in breast milk is thought to have a role in early neural development, ${ }^{10}$ and some studies have found that DHA may be associated with better cognitive outcome and higher IQ; however, further investigation is needed. ${ }^{32}$ Of note, evidence in humans indicated that $n-3$ EFA intake can elevate RvD1, RvD2, PD1, and 17-HDHA levels in healthy individuals. ${ }^{33}$ Increases in specific SPMs after $n-3$ EFA intake followed by aspirin are associated with enhanced functional outcome in whole blood (i.e., increased phagocytosis) demonstrating functional metabolomic profiling. ${ }^{3}$ Omega-3 intake elevated $\mathrm{RvD1}$ levels in diabetic mice ${ }^{34}$ and in patients with minor cognitive impairment and was associated with enhanced uptake of $\beta$-amyloid. ${ }^{35}$ AT-RvD1 improves postoperative cognitive decline in mice, ${ }^{36}$ and RvE1 and AT-RvD1 differentially improve functional outcome following diffuse traumatic brain injury. ${ }^{37}$ Hence, taken together with present findings that human milk contains a proresolving LM-SPM 
signature profile, human milk SPMs may be relevant in infant neurological development.

In summation, human milk LM metabololipidomic profiling uncovered specific LM signature with physiologically relevant levels of endogenous SPMs associated with accelerated resolution of acute inflammation in vivo. By profiling LMSPM in human milk, we identified several potent bioactive proresolving mediators including AT-RvD1, RvD2, RvD3, ATRvD3, RvD4, RvD5, RvD6, MaR1, PD1, AT-PD1, RvE2, RvE3, AT-LXA ${ }_{4}$, and $\mathrm{LXB}_{4}$ in human milk, as well as confirmed the earlier identification of RvD1, RvE1, and $\mathrm{LXA}_{4}{ }^{6}$ Mastitis milk had higher prostanoids, lower SPM, and reduced ability to accelerate resolution. Of these newly identified SPMs herein, RvD2 and MaR1 each accelerated resolution of acute inflammation and infection (Figure 4, Table 2, and Supplementary Figure 2). With human macrophages, HLMIs stimulate efferocytosis and containment of $E$. coli, key actions in resolution of inflammation and infection, and accelerate resolution of infection in vivo. Hence, the present results implicate a role for SPMs in modulating inflammation, infection, and stimulating resolution during early immune development, as SPMs display potent actions in the innate immune system.

\section{METHODS}

Extraction of HLMIs for murine peritonitis. Deidentified human milk from healthy donors was purchased from Biological Specialty Corporation (Colmar, PA) or from healthy and matched mastitis donors from Creative Bioarray (Shirley, NY). Two volumes of methanol were added to milk, and proteins were precipitated for $30 \mathrm{~min}$ on ice. Precipitate was pelleted by centrifugation $\left(10,000\right.$ r.p.m. at $4{ }^{\circ} \mathrm{C}$ for $10 \mathrm{~min})$. Supernatants were extracted using two volumes of diethyl ether, and LMs were further isolated using solid-phase extraction as in Colas et al. ${ }^{3}$ Products were eluted in methyl formate; solvent was evaporated under $\mathrm{N}_{2}$, and resuspended in ethanol. Aliquots of the ethanol fractions were taken to LC-MS-MS-based metabololipidomics for LM profiling.

Peritonitis and resolution indices. Sterile self-limited peritonitis was initiated in male FVB mice (6-8 weeks; Charles River Laboratories, Newton, MA) by intraperitoneal injection of $1 \mathrm{mg}$ zymosan A (Z4250; Sigma-Aldrich, St. Louis, MO) ${ }^{38}$ For infectious peritonitis, mice were injected with self-limited inoculum of $E$. coli $\left(10^{5}\right.$ colony-forming unit). Immediately before zymosan injection, mice were administered (intraperitoneally) HLMIs (levels representative of $\sim 1 \mathrm{ml}$ human milk), RvD2 (50 ng per mouse), MaR1 (50 ng per mouse), or vehicle (saline containing $0.2 \%$ ethanol). In some experiments, mice were administered treatments at $T_{\max }(12 \mathrm{~h})$. Isolates pooled from three human milk donors were used in determining the impact on the $R_{\mathrm{i}}$ of acute peritonitis. RvD2 and MaR1 for each experiment were prepared by total organic synthesis, and matched to authentic RvD2 and MaR1 ${ }^{20}$ Physical properties of RvD2 and MaR1 were validated before each experiment according to published criteria. ${ }^{20}$ Peritoneal exudates were collected at indicated time intervals by lavaging with $5 \mathrm{ml}$ PBS (phosphate-buffered saline). Exudate PMN numbers were assessed using Turk's solution, light microscopy, and flow cytometry (FACSCanto II; BD Bioscience, San Jose, CA). PMNs were determined as Ly6G- (clone 1A8; BD Bioscience) and CD11b- (clone M1/70; eBioscience, San Diego, CA) positive events and F4/80- (clone BM8; eBioscience) negative events from events as assessed by forward scatter and side scatter. Resolution indices were calculated as in Schwab et al. ${ }^{13}$ and Bannenberg et al., ${ }^{14}$ where $\Psi_{\max }$ is the maximal PMN count, $T_{\max }$ the time interval when PMN reaches maximum, $T_{50}$ the time interval corresponding to $50 \%$ PMN reduction (or $\Psi_{50}$ ), and the $R_{\mathrm{i}}$ is the interval between $T_{\max }$ and $T_{50}$. All animal experiments were approved by the Standing Committee on Animals of Harvard Medical School (protocol no. 02570) and performed in accordance with institutional guidelines.

LC-MS-MS-based LM metabololipidomics of human milk. For quantification of LM, human milk from four healthy donors (1-2 months postpartum; Lee Biosolutions, Maryland Heights, MO) or matched mastitis and healthy donors (1-6 months postpartum; Creative Bioarray, Shirley, NY) was extracted using solid-phase extraction with C18 columns (Waters, Milford, MA), following the addition of three volumes of cold methanol containing deuterated internal standards (1 ng d4-PGE 2 , d4-LTB4, and d8-5S-HETE, as well as d5-RvD2) and protein precipitation. LM levels were assessed by a LC-MS-MS system, QTrap 5500 and QTrap 6500 (ABSciex, Concord, Ontario, Canada) equipped with Shimadzu LC-20AD HPLC and a Shimadzu SIL-20AC autoinjector (Shimadzu, Kyoto, Japan). An Agilent Eclipse Plus C18 column $(100 \mathrm{~mm} \times 4.6 \mathrm{~mm} \times 1.8 \mu \mathrm{m})$ was used with a gradient of methanol/water/acetic acid of 55:45:0.01 ( $\left.\mathrm{vol} \mathrm{vol}^{-1} \mathrm{vol}^{-1}\right)$ to 100:0:0.01 at $0.4 \mathrm{ml} \mathrm{min}{ }^{-1}$ flow rate. To monitor and identify various LM, a multiple reaction monitoring method was developed with signature ion pairs, Q1 (parent ion)-Q3 (characteristic daughter ion) optimized for each molecule. Identification was conducted using published criteria, ${ }^{3}$ where a minimum of six diagnostic ions were used in each MS-MS. The complete stereochemistry of resolvin D4 was recently determined, ${ }^{39}$ and the synthetic standard was used here for identification and quantitation from human milk. Linear calibration curves for each compound were obtained with $r^{2}$ values ranging from 0.98 to 0.99 . Detection limits were $\sim 0.1 \mathrm{pg}$.

Principal component analysis. Principal component analysis was performed using SIMCA 13.0.3 software (Umetrics, San Jose, CA) following mean centering and unit variance scaling of LM amounts. Principal component analysis is an unbiased, multivariate projection designed to identify the systematic variation in a data matrix (the overall bioactive LM profile of each sample) with lower dimensional plane using score plots and loading plots. The score plot shows the systematic clusters among the observations (closer plots presenting higher similarity in the data matrix). Loading plots describe the magnitude and the manner (positive or negative correlation) in which the measured LMs/SPMs contribute to the cluster separation in the score plot. ${ }^{40}$

Depletion of milk LMs using activated charcoal adsorption. Human milk from three healthy donors $(10 \mathrm{ml}$ from each donor) was combined and incubated with or without $4 \%$ activated charcoal (Sigma) for $1 \mathrm{~h}$ at room temperature. Activated charcoal was washed out, three volumes methanol were added to the milk, and proteins precipitated at $-20^{\circ} \mathrm{C}$. Precipitate was pelleted by centrifugation (3,000 r.p.m. at $4{ }^{\circ} \mathrm{C}$ for $10 \mathrm{~min}$ ), and LMs isolated using C18 columns and solid-phase extraction. ${ }^{3}$ Products were eluted in methyl formate, solvent was evaporated under $\mathrm{N}_{2}$, and suspended in $500 \mu$ lethanol. For human macrophage phagocytosis, $20 \mu \mathrm{l}$ HLMIs were dried down and resuspended in $1 \mathrm{ml} \mathrm{PBS}^{+1+}$ (highest dilution =1) followed by indicated dilutions (10-1,000-fold). Aliquots of the ethanol fractions were taken to LC-MS-MS-based metabololipidomics for quantification of LM profiling.

Human macrophage phagocytosis and efferocytosis. To obtain apoptotic PMN, human PMNs were isolated by density-gradient Ficoll-Histopaque from human peripheral blood. Blood was obtained from healthy volunteers giving informed consent according to Partners Human Research Committee Protocol no. 1999-P-001297. PMNs were labeled with Bisbenzimide H 33342 (Sigma-Aldrich), a fluorescent nuclear dye $\left(10 \mu \mathrm{g} \mathrm{ml}{ }^{-1}\right.$ for $30 \mathrm{~min}$ at $\left.37^{\circ} \mathrm{C}\right)$ and cultured overnight $\left(5 \times 10^{6}\right.$ cells per $\mathrm{ml}$ in $\left.\mathrm{PBS}^{+/+}\right)$. Human primary macrophages were differentiated from peripheral blood monocytes ${ }^{19}$ and plated onto 96 -well plates $\left(5 \times 10^{4}\right.$ cells per well $)$. Macrophages were 
incubated with either HLMIs or $\mathrm{HLMI}_{\mathrm{AC}}$ at indicated dilutions (1-1,000 fold dilutions, $\mathrm{pH} 7.45$, at $37^{\circ} \mathrm{C}$ for $15 \mathrm{~min}$ ), followed by a phagocytic challenge with either fluorescently labeled apoptotic PMN (3:1 PMN:macrophage) or E. coli (50:1 E. coli:macrophage). Incubations were continued for $45 \mathrm{~min}$ at $37{ }^{\circ} \mathrm{C},{ }^{19}$ macrophages washed, and remaining extracellular fluorescence quenched using Trypan Blue (1:15 Trypan blue:PBS $\left.{ }^{+/+}\right)$. Phagocytosis was assessed using a SpectraMax M3 plate reader (Molecular Devices, Sunnyvale, CA).

Statistics. Data are presented as individual values or mean \pm s.e.m. The criterion for statistical significance was $P<0.05$ using nonparametric Mann-Whitney test or two-way analysis of variance, followed by a post hoc Bonferroni test using GraphPad Prism 6 (La Jolla, CA).

SUPPLEMENTARY MATERIAL is linked to the online version of the paper at http://www.nature.com/mi

\section{ACKNOWLEDGMENTS}

We thank Mary Halm Small for expert assistance in the manuscript preparation and lliyan Vlasakov for technical assistance. This work was supported in part by NIH grant P01GM095467 (CNS) and a research grant from Solutex (Madrid, Spain; to CNS). HA was supported by an Arthritis Foundation Postdoctoral Fellowship Award. SKO was supported by a Canadian Institutes of Health Research Fellowship Award.

\section{DISCLOSURE}

CNS is an inventor on patents (resolvins) assigned to BWH and licensed to Resolvyx Pharmaceuticals. CNS is a scientific founder of Resolvyx Pharmaceuticals and owns equity in the company. CNS' interests were reviewed and are managed by the Brigham and Women's Hospital and Partners HealthCare in accordance with their conflict of interest policies. The remaining authors declare no conflicts of interest.

c) 2016 Society for Mucosal Immunology

\section{REFERENCES}

1. Serhan, C.N. Pro-resolving lipid mediators are leads for resolution physiology. Nature 510, 92-101 (2014).

2. Fullerton, J.N., O'Brien, A.J. \& Gilroy, D.W. Lipid mediators in immune dysfunction after severe inflammation. Trends Immunol. 35, 12-21 (2014).

3. Colas, R.A., Shinohara, M., Dalli, J., Chiang, N. \& Serhan, C.N. Identification and signature profiles for pro-resolving and inflammatory lipid mediators in human tissue. Am. J. Physiol. Cell. Physiol. 307, C39-C54 (2014).

4. Claria, J., Nguyen, B.T., Madenci, A., Ozaki, C.K. \& Serhan, C.N. Diversity of lipid mediators in human adipose tissue depots. Am. J. Physiol. Cell. Physiol. 304, C1141-C1149 (2013).

5. Keelan, J.A. et al. Effects of maternal n-3 fatty acid supplementation on placental cytokines, pro-resolving lipid mediators and their precursors. Reproduction 149, 171-178 (2015).

6. Weiss, G.A., Troxler, H., Klinke, G., Rogler, D., Braegger, C. \& Hersberger, M. High levels of anti-inflammatory and pro-resolving lipid mediators lipoxins and resolvins and declining docosahexaenoic acid levels in human milk during the first month of lactation. Lipids Health Dis. 12, 89 (2013).

7. Serhan, C.N. et al. Reduced inflammation and tissue damage in transgenic rabbits overexpressing 15-lipoxygenase and endogenous anti-inflammatory lipid mediators. J. Immunol. 171, 6856-6865 (2003).

8. Arita, M. etal. Resolvin E1, an endogenous lipid mediator derived from omega3 eicosapentaenoic acid, protects against 2,4,6-trinitrobenzene sulfonic acidinduced colitis. Proc. Natt. Acad. Sci. USA 102, 7671-7676 (2005).

9. Bento, A.F., Claudino, R.F., Dutra, R.C., Marcon, R. \& Calixto, J.B. Omega3 fatty acid-derived mediators 17(R)-hydroxy docosahexaenoic acid, aspirin-triggered resolvin D1 and resolvin D2 prevent experimental colitis in mice. J. Immunol. 187, 1957-1969 (2011).

10. Calder, P.C. et al. Early nutrition and immunity—progress and perspectives. Br. J. Nutr. 96, 774-790 (2006).

11. Peng, Y. et al. Fatty acid composition of diet, cord blood and breast milk in Chinese mothers with different dietary habits. Prostaglandins Leukot. Essent. Fatty Acids 81, 325-330 (2009).
12. Walker, W.A. Initial intestinal colonization in the human infant and immune homeostasis. Ann. Nutr. Metab. 63 (Suppl 2), 8-15 (2013).

13. Schwab, J.M., Chiang, N., Arita, M. \& Serhan, C.N. Resolvin E1 and protectin D1 activate inflammation-resolution programmes. Nature 447, 869-874 (2007).

14. Bannenberg, G.L. et al. Molecular circuits of resolution: formation and actions of resolvins and protectins. J. Immunol. 174, 4345-4355 (2005).

15. Reid, B., Smith, H. \& Friedman, Z. Prostaglandins in human milk. Pediatrics 66, 870-872 (1980).

16. Levy, B.D., Clish, C.B., Schmidt, B., Gronert, K. \& Serhan, C.N. Lipid mediator class switching during acute inflammation: signals in resolution. Nat. Immunol. 2, 612-619 (2001).

17. Flower, R.J. Prostaglandins, bioassay and inflammation. Br. J. Pharmacol. 147, S182-S192 (2006)

18. Haeggstrom, J.Z. \& Funk, C.D. Lipoxygenase and leukotriene pathways: biochemistry, biology, and roles in disease. Chem. Rev. 111, 5866-5898 (2011).

19. Chiang, N. et al. Infection regulates pro-resolving mediators that lower antibiotic requirements. Nature 484, 524-528 (2012).

20. Spite, M. et al. Resolvin D2 is a potent regulator of leukocytes and controls microbial sepsis. Nature 461, 1287-1291 (2009).

21. Chen, R.F. Removal of fatty acids from serum albumin by charcoal treatment. J. Biol. Chem. 242, 173-181 (1967).

22. Chiang, N., Dalli, J., Colas, R.A. \& Serhan, C.N. Identification of resolvin D2 receptor mediating resolution of infections and organ protection. J. Exp. Med. 212, 1203-1217 (2015).

23. Gronert, K., Gewirtz, A., Madara, J.L. \& Serhan, C.N. Identification of a human enterocyte lipoxin A4 receptor that is regulated by $\mathrm{IL}-13$ and IFN-gamma and inhibits TNF-alpha-induced IL-8 release. J. Exp. Med. 187, 1285-1294 (1998).

24. Gewirtz, A.T. et al. Pathogen-induced chemokine secretion from model intestinal epithelium is inhibited by lipoxin A4 analogs. J. Clin. Invest. 101, 1860-1869 (1998).

25. Campbell, E.L. et al. Resolvin E1-induced intestinal alkaline phosphatase promotes resolution of inflammation through LPS detoxification. Proc. Natl. Acad. Sci. USA 107, 14298-14303 (2010).

26. Morita, M. et al. The lipid mediator protectin D1 inhibits influenza virus replication and improves severe influenza. Cell 153, 112-125 (2013).

27. Aitken, S.L., Corl, C.M. \& Sordillo, L.M. Immunopathology of mastitis: insights into disease recognition and resolution. J. Mammary Gland Biol. Neoplasia 16, 291-304 (2011).

28. Marcon, R., Bento, A.F., Dutra, R.C., Bicca, M.A., Leite, D.F. \& Calixto, J.B. Maresin 1, a proresolving lipid mediator derived from omega-3 polyunsaturated fatty acids, exerts protective actions in murine models of colitis. J. Immunol. 191, 4288-4298 (2013).

29. Recchiuti, A. et al. Immunoresolving actions of oral resolvin D1 include selective regulation of the transcription machinery in resolution-phase mouse macrophages. FASEB J. 28, 3090-3102 (2014).

30. World Health Organization. Long-Term Effects of Breastfeeding: A Systematic Review. Available athttp://www.who.int/maternal_child_adolescent/documents/breastfeeding_long_term_effects/en/2013.

31. Fidler, N., Sauerwald, T., Pohl, A., Demmelmair, H. \& Koletzko, B. Docosahexaenoic acid transfer into human milk after dietary supplementation: a randomized clinical trial. J. Lipid Res. 41, 1376-1383 (2000).

32. Heaton, A.E., Meldrum, S.J., Foster, J.K., Prescott, S.L. \& Simmer, K. Does docosahexaenoic acid supplementation in term infants enhance neurocognitive functioning in infancy? Front. Hum. Neurosci. 7, 774 (2013).

33. Mas, E., Croft, K.D., Zahra, P., Barden, A. \& Mori, T.A. Resolvins D1, D2, and other mediators of self-limited resolution of inflammation in human blood following n-3 fatty acid supplementation. Clin. Chem. 58, 1476-1484 (2012).

34. Shevalye, H. et al. Effect of enriching the diet with menhaden oil or daily treatment with resolvin D1 on neuropathy in a mouse model of type 2 diabetes. J. Neurophysiol. 114, 199-208 (2015).

35. Fiala, M. et al. Omega-3 supplementation increases amyloid-beta phagocytosis and resolvin D1 in patients with minor cognitive impairment. FASEB J. 29, 2681-2689 (2015).

36. Terrando, N. et al. Aspirin-triggered resolvin D1 prevents surgery-induced cognitive decline. FASEB J. 27, 3564-3571 (2013). 


\section{ARTICLES}

37. Harrison, J.L. et al. Resolvins AT-D1 and E1 differentially impact functional outcome, post-traumatic sleep, and microglial activation following diffuse brain injury in the mouse. Brain Behav. Immun. 47, 131-140 (2015).

38. Sampaio, A.L.F., Dufton, N. \& Perretti, M. Models of acute inflammationair pouch, peritonitis, and ischemia-reperfusion. In Fundamentals of Inflammation (Serhan, C.N. \& Ward, P.A. \& Gilroy, D.W., eds) 329-337 (Cambridge University Press, New York, NY, 2010).
39. Winkler, J. et al. Resolvin D4 potent antiinflammatory proresolving actions confirmed via total synthesis. FASEB J. 29, Suppl, 285.10 (2015).

40. Janes, K.A. \& Yaffe, M.B. Data-driven modelling of signal-transduction networks. Nat. Rev. Mol. Cell Biol. 7, 820-828 (2006).

41. Arnardottir, H.H., Dalli, J., Colas, R.A., Shinohara, M. \& Serhan, C.N. Aging delays resolution of acute inflammation in mice: reprogramming the host response with novel nano-proresolving medicines. J. Immunol. 193, 4235-4244 (2014). 